Perez, C. A., Kaiman, H. A., Keith, J., Mill, W. B., Vietti, T. J., and Powers, W. E. (1973). Treatment of Wilms' tumor and factors affecting prognosis. Cancer, 32, 609-617 .

Paul C. Rogers, Betty J. Wood, David F. Smith, AND J. M. TEASDALE

Departments of Paediatrics and Diagnostic Radiology, University of British Columbia, Vancouver, British Columbia, Canada V5Z 1 M9

Correspondence to Dr P. C. J. Rogers, Department of Paediatrics, University of British Columbia, 715 West 12th Avenue, Vancouver, British Columbia, Canada V5Z 1 M9.

\section{Thermometers and rectal perforations in the neonate}

Spontaneous perforation of the gastrointestinal tract in the neonate is rare. When it occurs it usually involves the stomach or the ileocaecal region. Perforation of the rectum in the neonate is usually caused by instruments being passed into it. Two patients with neonatal rectal perforations, probably caused by thermometers, have been admitted to the hospital during the last 6 months. Only one other case could be found from our records during the last 15 years.

\section{Case reports}

Case 1. A baby boy, birthweight $4.5 \mathrm{~kg}$, term, forceps delivery for delay in the 2nd stage of labour. The abdomen was noted to be mildly distended at birth. At age 7 hours, while the baby's temperature was being taken rectally, the thermometer snapped. The abdomen became more distended and an erect $x$-ray showed free gas in the peritoneal cavity. At laparotomy on the same day, there was copious peritoneal fluid and the small bowel was matted together with fibrin. There was a $4 \mathrm{~mm}$ perforation in the anterior rectal wall. The perforation was oversewn and a double barrel transverse colostomy performed. The baby's recovery was uneventful and the colostomy was closed 6 months later.

Case 2. A baby girl, birthweight $2.6 \mathrm{~kg}$, term, normal delivery. She was well until 4 days old when she suddenly became ill with circulatory collapse and marked abdominal distension. An erect $x$-ray of the abdomen showed free gas in the peritoneal cavity. After resuscitation, at laparotomy, there was gross faecal peritonitis. The rectum was contused for a distance of $2 \mathrm{~cm}$ above the peritoneal reflection and there were 2 perforations in the anterior wall of the rectum. The cause of this damage was unknown at the time of operation so the contused segment was excised, the rectum oversewn, and the sigmoid colon brought out as an end colostomy. Histological examination of the resected specimen confirmed the severe contusions and haemorrhage but the mucosa was found to be undamaged except for the perforations. The postoperative course was complicated by a wound dehiscence but was otherwise satisfactory and the patient is now awaiting a rectosigmoid anastamosis.

Case 3. A baby boy, birthweight $2.9 \mathrm{~kg}$, term, normal delivery. On the 2 nd day of life, having passed meconium and taken his feeds well, he began to vomit bile-stained material and the abdomen became distended. An erect $x$-ray of the abdomen showed free gas in the peritoneal cavity. He was resuscitated and at laparotomy there was a large amount of turbid fluid in the peritoneal cavity. After an extensive search a perforation, $5 \mathrm{~mm}$ in diameter, was found in the anterior rectal wall at the level of the peritoneal reflection. The perforation was oversewn and a loop sigmoid colostomy performed. He made an uneventful recovery and the colostomy was closed 6 weeks later.

\section{Discussion}

As with most previous reports it was not always easy to prove that a thermometer was the cause of the rectal perforation. In Case 1 the thermometer actually snapped inside the baby's rectum. Cases 2 and 3 had been transferred from different maternity units where it was normal practice to measure temperatures rectally. Although the actual incident causing the perforation could not be identified the lesions were characteristic. The presence of a small circumscribed perforation in the anterior rectal wall at the level of the peritoneal reflection, in a baby transferred from a hospital where the rectal thermometer is routinely used, is sufficient to implicate the procedure. A 4th baby has been admitted to this hospital with clinical and laparotomy findings similar to those reported above. Although the lesion appeared to be the result of instrumentation of the rectum, the baby had been born at home and no evidence of the insertion of a thermometer into the rectum could be obtained.

Neonatal rectal perforations have been observed after the passing of various types of instruments, such as catheters used in rectal washouts and barium 
enema examinations. The rectal thermometer was first implicated by Segnitz (1957) and since then a further 10 patients have been reported. Greenbaum et al. (1969) found a $70 \%$ mortality in this condition and, taking into account the few more recent cases, the mortality still approaches $50 \%$. One reason for this high mortality is that a small rectal perforation is often difficult to find at laparotomy. The perforation in Case 3 was found only with difficulty and, in the patient reported by Segnitz (1957), the perforation was missed and only found at necropsy. It is important therefore, in any baby with a pneumoperitoneum, to examine the rectum carefully if no other site of a perforation has been found.

Smiddy and Benson (1969) pointed out that the shape of the neonatal rectum causes a thermometer to impinge on the anterior wall when it is inserted to a depth of $2 \mathrm{~cm}$ and any attempt to push it further may result in perforation. Merenstein (1970), stated that the thermometer needs to be inserted to at least $5 \mathrm{~cm}$ to get an adequate core temperature. These arguments would seem to be superfluous, as Tsingoglou and Wilkinson (1971) showed that the closed axillary temperature correlates well with the rectal temperature and that if there should be any difference it would be no more than $0 \cdot 2^{\circ} \mathrm{C}$.

We therefore agree with the editorial in British Medical Journal (1970) that the only way to eradicate this potentially fatal condition is to stop 'unhygienic, unnecessary, and dangerous' use of the rectal thermometer in the neonate.

\section{Summary}

Three neonates with rectal perforations, probably caused by the use of the rectal thermometer, are reported. The dangers of this condition and the difficulty of diagnosis even at laparotomy are emphasised. It is suggested that since the axillary temperature in the neonate is an adequate reflection of the core temperature the routine use of the rectal thermometer, except in exceptional circumstances, should cease.

We thank Professor A. W Wilkinson for permission to report these patients

\section{References}

British Medical Journal (1970). Editorial: Hazards of temperature taking. British Medical Journal, 3, 4-5.

Greenbaum, E. I., Carson, M., Kimcammon, W. N., and O'Loughlin, B. J. (1969). Rectal thermometer induced pneumoperitoneum in the newborn. Pediatrics, 44, 539-542.
Merenstein, G. B. (1970). Letter: Rectal perforation by thermometer. Lancet, 1, 1007.

Segnitz, R. M. (1957). Accidental transanal perforation of the rectum. American Journal of Diseases of Children, 93, 255-258.

Smiddy, F. G., and Benson, E. A. (1969). Rectal perforation by thermometer. Lancet, 2, 805-806.

Tsingoglou, S., and Wilkinson, A. W. (1971). Heat loss during neonatal operations. Archives of Disease in Childhood, 46, 452-456.

\section{J. D. Frank AND S. Brown}

The Hospital for Sick Children, Great Ormond Street, London WC1N $3 \mathrm{JH}$

Correspondence to J. D. Frank FRCS.

\section{Editorial comment}

Four paediatricians, with much neonatal experience, Dr P. M. Dunn (Bristol), Dr J. M. Parkin (Newcastle), Professor J. Scopes (London), and Dr N. R. C. Roberton (Cambridge), were asked to comment on this report and to give their views on when, and how, the temperature of (a) normal newborn babies, and (b) preterm, and other babies in special care, should be taken.

(a) Normal babies. 3 of the 4 agreed that the babies' temperature should be taken on arrival from the delivery room to the lying-in ward. Thereafter some continued to take temperatures once or twice daily, others only if some indication was present. An ordinary glass thermometer is used rectally in two units and in the axilla in two.

(b) Preterm and special care babies. All agreed on the importance of regular thermometry, with the frequency depending on the individual case. While rectal thermometers (glass or electric) are still often used, continuous recording of skin (or axillary) temperature is much favoured.

Dangers. Breaking of glass thermometers in the rectum was an occasional accident, although this did not necessarily lead to the dire results reported. Infections had occasionally been traced to rectal thermometers.

Teaching. There does not seem to be an established technique taught to nurses on how a rectal thermometer should be inserted to avoid trauma.

A useful discussion of these matters is on pp. 105-106 of Medical Care of Newborn Babies (1972) by Davies, Robinson, Scopes, Tizard, and Wigglesworth. 